\title{
Biaxially oriented CdSe nanorods
}

Dag W. Breiby', Patrick T.K. Chin', Jens W. Andreasen ${ }^{3}$, Kim A. Grimsrud', Zhenyu Di ${ }^{4}$, René A.J. Janssen $^{2}$

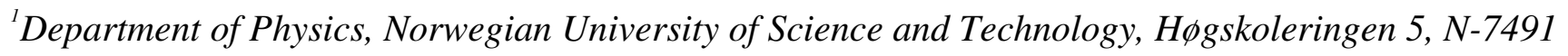
Trondheim, Norway

${ }^{2}$ Molecular Materials and Nanosystems, Eindhoven University of Technology, PO Box 513, NL-5600 MB Eindhoven, The Netherlands

${ }^{3}$ The Solar Energy Programme, Ris $\phi$ National Laboratory for Sustainable Energy, Technical University of Denmark - DTU, P.O. Box 49, DK-4000 Roskilde, Denmark

${ }^{4}$ Department of Physics, Technical University of Munich, James-Franck-Strasse 1, D-85747 Garching, Germany

\begin{abstract}
The shape, structure, and orientation of rubbing-aligned cadmium selenide (CdSe) nanorods on polymer coated glass substrates have been studied using TEM and grazing incidence X-ray scattering combined with computer simulations. The nanorods are found to be of wurtzite structure and highly monodisperse, and have an essentially ellipsoidal shape with short axes $\sim 8 \mathrm{~nm}$ and long axis $\sim 22 \mathrm{~nm}$. The nanorods exhibit preferred biaxial orientation with the hexagonal $a$-c-plane parallel to the sample surface, and the $c$-axis oriented along the rubbing direction of the sample. Some tendency of smectic-A ordering is observed. A quantitative model incorporating atomic structure, rod shape, and preferred orientation was developed for numerically simulating the diffraction peak positions, widths and intensities, giving good correlation with the experimental observations.
\end{abstract}


Keywords: nano particles; CdSe; TEM; GISAXS; X-ray diffraction; simulations.

Nanoparticles represent a very active area of materials science because of their interesting electronic properties relating to quantum confinement and their high ratio of surface-near atoms. ${ }^{1-4}$ Numerous applications are emerging in such diverse fields as cosmetics, solar cells, electronics, light emitting diodes, catalysis, and biological markers. ${ }^{5-9}$ Nanoparticles can be processed to different shapes, ranging from spheres and ellipsoids to, e.g., tri- and tetrapods, and regular nanowires. ${ }^{2,10-12}$ During the last two decades, there has been a strong interest in cadmium chalcogenide nanoparticles. CdSe nanoparticles in particular have been used as a testing system for studies of nanoscale electronic, optical, optoelectronic, and chemical processes. ${ }^{2,10,13}$ It has been reported that CdSe nanorods can be macroscopically aligned and give polarization dependent optical properties, which can be an advantage in the development of polarized light sources. ${ }^{14-16}$ Macroscopic crystals of CdSe have two dominant structures, wurtzite (space group $\mathrm{P}_{3} \mathrm{mc}$ ) and zinc blende (space group $\left.\mathrm{F} \overline{4} 3 \mathrm{~m}\right){ }^{17}$ Some earlier studies indicate that CdSe nanoparticles are best described as a mixture of these structures. ${ }^{2}$ Under high-pressure conditions, the bulk structure of CdSe transforms into the rock salt structure followed by the orthorhombic $\mathrm{Cmcm}$ phase with further increasing pressure. Similar transformations take place at higher pressure in CdSe nanoparticles. $^{18}$

For an improved understanding of the physicochemical properties of nanoparticles, accurate determination of crystal structure, strain effects, shape, size and orientational distributions is desired. To our knowledge, the complete atomic structure has only been obtained for a few Au-based nanoparticle complexes, ${ }^{4,19,20}$ by X-ray diffraction of single crystals grown from a large number of identical nanoparticles. It is obvious that despite the great results obtained, this method suffers from severe limitations, mainly relating to sample preparation. Hence, coherent diffractive imaging, as recently demonstrated, appears to be a more generally viable path for future structure investigations, ${ }^{21,}{ }^{22}$ but suffers from the need for highly specialized equipment. Established crystallography methods are not always applicable to independent particles in the nanometer-range, where the assumption of "infinite" 
crystal sizes breaks down. Determination of nanoparticle structures thus demands techniques that are better suited for ultra-small $(<100 \mathrm{~nm})$ crystals. With the rapidly increasing computing power available, calculations of the scattering from increasingly larger systems, taking every atomic coordinate into account, are nowadays feasible.

Here, we present a combined TEM and X-ray scattering study of the crystal structure and orientation of CdSe nanorods that have been spin-coated onto a thin film of the conjugated polymer poly $(3,4-$ ethylenedioxythiophene):polystyrenesulfonic acid (PEDOT:PSS) and subsequently rubbed. (Nonrubbed samples were not studied). Grazing-incidence wide- and small-angle X-ray scattering (GIWAXS/GISAXS) have been performed, which provides orientational information in thin films ${ }^{21,23-27}$ in addition to the structural information reported from $\mathrm{TEM}^{2}$ and traditional powder X-ray diffraction. ${ }^{10}$ ${ }^{28,29}$ (See Supporting Information for full experimental details). Of related interest to this work are the previous GIWAXS studies on CdSe, Refs. ${ }^{30,31}$, and on biaxial orientation by Knaapila et al. ${ }^{32}$. We devise a simplest possible model to account for the experimental data, including the crystal structure with disorder, the external particle shape and biaxial orientation distributions. Computer simulated Xray diffraction patterns based on this model compare well with the experimental results.

GIWAXS and GISAXS are established techniques for non-destructive investigations of nanostructures in thin films, ${ }^{23,33,34}$ which are receiving steadily increasing attention for their capability of measuring crystallinity, atomic structures, and domain orientations. These techniques rely on the fact that the refractive index for X-rays is slightly less than unity, thus giving total external reflection for a grazing incidence angle $\alpha_{\mathrm{i}}$ smaller than a material-specific critical angle $\alpha_{\mathrm{c}}$, typically about $0.2^{\circ} .^{23}$ The incoming and outgoing monochromatic X-ray beams are specified by the wave vectors $\mathbf{k}_{\mathrm{i}}$ and $\mathbf{k}_{\mathrm{f}}$, respectively. The scattering is elastic, and the scattering vector is $\mathbf{Q}=\mathbf{k}_{\mathrm{f}}-\mathbf{k}_{\mathrm{i}}$, with magnitude $Q=$ $4 \pi \sin \theta / \lambda$, where $\theta$ is half the scattering angle and $\lambda$ the wavelength. The Laue condition for diffraction is $\mathbf{Q}=\mathbf{G}$, where $\mathbf{G}$ is a reciprocal lattice vector. The scattered intensity $I$ for a coherently scattering system containing $j$ atoms is given by $I=|F|^{2}$, where the scattering amplitude $F$ is given by

$$
F=\sum_{j} f_{j}(\mathbf{Q}) \exp \left(i \mathbf{Q} \cdot \mathbf{r}_{j}\right)
$$


The sum is over all atoms in the nanoparticle, $f_{\mathrm{j}}(\mathbf{Q})$ the atomic form factor, and $\mathbf{r}_{\mathrm{j}}$ the position vector of the $j^{\text {th }}$ atom. Since (1) contains the directions of the vectors $\mathbf{Q}$ and $\mathbf{r}$ it can be applied to a single crystal with a defined orientation.

For an ensemble of particles exhibiting preferred orientation, we model the scattered intensities as an incoherent sum of intensities from the individual nanoparticles. In recent works ${ }^{24,35}$,(See also Supporting Information) we developed a formalism in which pseudo-Voigt weighting functions $\mathrm{P}_{\mathrm{i}}\left(W_{\mathrm{i}}, \Delta_{\mathrm{i}}, \mu_{\mathrm{i}}\right)$ account for radial peak broadening (in $Q$, distribution $\mathrm{P}_{\mathrm{Q}}$ ) and preferred orientation (functions $\mathrm{P}_{\chi}$ and $\left.\mathrm{P}_{\varphi}\right)$. The first argument $W_{\mathrm{i}}$ is a measure of the distribution width, the second $\Delta_{\mathrm{i}}$ represents the offset from the peak maximum, and $\mu_{\mathrm{i}} \in(0,1)$ is the pseudo-Voigt weighting parameter governing the peak shape. $\mathrm{P}_{\chi}$ and $\mathrm{P}_{\varphi}$ give distributions in the out-of-plane $(\chi)(\mathbf{Q}$ perpendicular to substrate) and in-plane $(\varphi)$ directions, respectively. An expression for the intensity then reads

$$
I_{j}\left(Q_{j}, \chi_{j}, \varphi_{j}\right) \propto \sum_{n} \mathrm{~L}\left|F_{h k l}\right|^{2} \mathrm{P}_{Q}\left(W_{Q}, Q_{j}-Q_{n}, \mu_{Q}\right) \mathrm{P}_{\chi}\left(W_{\chi}, \chi_{j}-\chi_{n}, \mu_{\chi}\right) \mathrm{P}_{\varphi}\left(W_{\varphi}, \varphi_{j}-\varphi_{n}, \mu_{\varphi}\right)
$$

$I_{\mathrm{j}}$ denotes the intensity at a given point in reciprocal space having spherical coordinates $\left(Q_{\mathrm{j}}, \chi_{\mathrm{j}}, \varphi_{\mathrm{j}}\right)$, and the sum gives a weighted contribution to $I_{\mathrm{j}}$ from all reciprocal lattice points $n$. L contains the various geometric corrections, and takes the form

$$
\mathrm{L}=\frac{\cos \theta}{\sin 2 \theta} \cos ^{2} 2 \theta \frac{1}{\sin 2 \theta} \frac{1}{\sin 2 \theta}
$$

Here, $2 \theta$ is the total scattering angle, the first factor is a general prefactor for powder diffraction, ${ }^{36}$ the second is the polarization factor for scattering in the horizontal plane at a linearly-polarized synchrotron, and the $3^{\text {rd }}$ and $4^{\text {th }}$ factors are the Lorentz and footprint area corrections, respectively. ${ }^{37}$ In our previous work, the width $W_{\mathrm{Q}}$ assigned to $\mathrm{P}_{\mathrm{Q}}$ accounts for both instrumental resolution and (isotropic) sample related peak broadening. In the present work, however, the $\delta$-shaped reciprocal lattice points associated with an infinite lattice are replaced by finite-extension regions with an intensity distribution obeying (1). Thus, here, $\mathrm{P}_{\mathrm{Q}}$ accounts for the instrumental broadening only, whereas (anisotropic) peak broadening caused by small particle dimensions is inherent in the formalism. We note that the 
approximation of (2) works well for relatively narrow orientation distributions $\left(W_{\chi}\right.$ and $W_{\varphi}$ small), but will be increasingly poor for particles with high aspect ratios and broad orientation distributions.

Our simulation procedure thus consists of three steps. i) A nanorod is built up atom by atom, according to either the wurtzite or the zinc blende structure, with lattice constants taken from Ref. ${ }^{17}$. Disorder at the atomic scale is accounted for by adding Gaussian-distributed random offset values $u$ with variance $\sigma$ to each atomic position coordinate $x, y, z$. The same values $\sigma$ were used for both $\mathrm{Cd}$ and Se, as the difference between the Debye-Waller factors for II-VI compounds is thought to be small. ${ }^{38}$ ii) An intensity distribution in reciprocal space is established. To this end, we discretize reciprocal space using a cubic grid with step length $\Delta Q=0.02 \AA^{-1}$, and calculate the structure factor squared in each node using (1). Nodes having less than $2 \%$ of the intensity in the node with the highest intensity are deleted to speed up calculations. iii) Having established the intensity distribution associated with a single nanoparticle in reciprocal space, various scans for a whole ensemble of particles can be calculated using (2) (See also Supporting Information).

It is instructive to consider the scattering from a nanoparticle in terms of the product of an infinite atomic crystal lattice $L$ and a particle shape factor $S ; L \times S$. By the convolution theorem, the Fourier transform $\mathscr{F}$ of the particle structure gives $\mathscr{F}(L \times S)=\mathscr{F}(L) \otimes \mathscr{F}(S)$. Thus, the Fourier transform of the particle shape will be repeated in every reciprocal lattice point. Considering an $S$ describing a prolate spheroid $\left(r_{\mathrm{a}}=r_{\mathrm{b}}<r_{\mathrm{c}}\right)$, the reciprocal space intensity distribution comprises approximately oblate spheroids centred at each reciprocal lattice point. Neglecting strain, the observed peak width $\Delta Q$ can be parameterized in terms of an effective correlation length $\xi$ by means of the Scherrer formula, reading ${ }^{39} \xi$ $=0.9 \times 2 \pi / \Delta Q$.

CdSe nanorods were spin coated onto a thin PEDOT:PSS film coated on a glass substrate. Then, the sample was rubbed gently with a velvet cloth to align the nanorods. For detailed description of the sample preparation, see Ref. ${ }^{16}$. In the subsequent sections, we demonstrate how the partially overlapping, yet complementary, characterization methods of TEM, GISAXS and GIWAXS are used to draw conclusions about the particle shapes, orientations, crystal structure, and intercorrelations. 


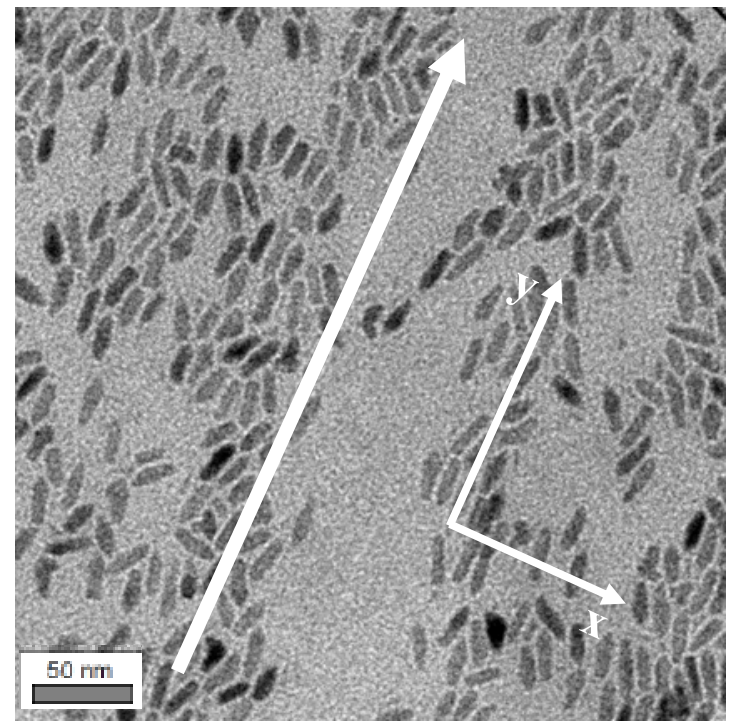

Figure 1. Bright-field TEM image of a rubbed CdSe nanorods film. Note that the rods are rather monodisperse, and tend to orient parallel to the rubbing direction. The contrast variations are ascribed to different orientations of the nanorods about their long axis. A coordinate system is defined for the X-ray diffraction measurements.

TEM provided clear evidence that the nanorods are nearly monodisperse, and that they tend to orient with the long axis along the rubbing direction, cf. Fig. 1. The particles have a rodlike shape, and we estimate a typical diameter to $80 \AA$ and length to $220 \AA$, which gives an aspect ratio of 0.4 . Note that some of the particles seen in Fig. 1 appear darker than others, which we ascribe to different orientations of the nanorods about their long axis. Particles having more strongly diffracting orientations will appear darker, and vice versa. The particles seem to have a preferred orientation with respect to the substrate, as a majority of them have approximately the same contrast. Moreover, they exhibit a similar rounded rod shape regardless of the different facet orientations, suggesting that the cross section is circular. By careful inspection of Fig. 1, it can be deduced that the particles show a tendency of smectic A ordering, as reported previously by Alivisatos et al. ${ }^{40}$

The GISAXS experiments are consistent with particles exhibiting preferred orientation (See Supporting Information). For relating the data to the TEM observations, we note by the mentioned Scherrer formula that the characteristic axes lengths of $80 \AA$ and $220 \AA$ correspond to $\sim 0.07 \AA^{-1}$ and 
$\sim 0.03 \AA^{-1}$, respectively. These values are in agreement with the GISAXS images, as the fwhm of the scattered intensity is comparable to these values. Since GISAXS gives an ensemble average over a large area $\left(\sim \mathrm{mm}^{2}\right)$, this suggests that the highly local TEM image shown in Fig. 1 is indeed representative for bigger areas of the sample.
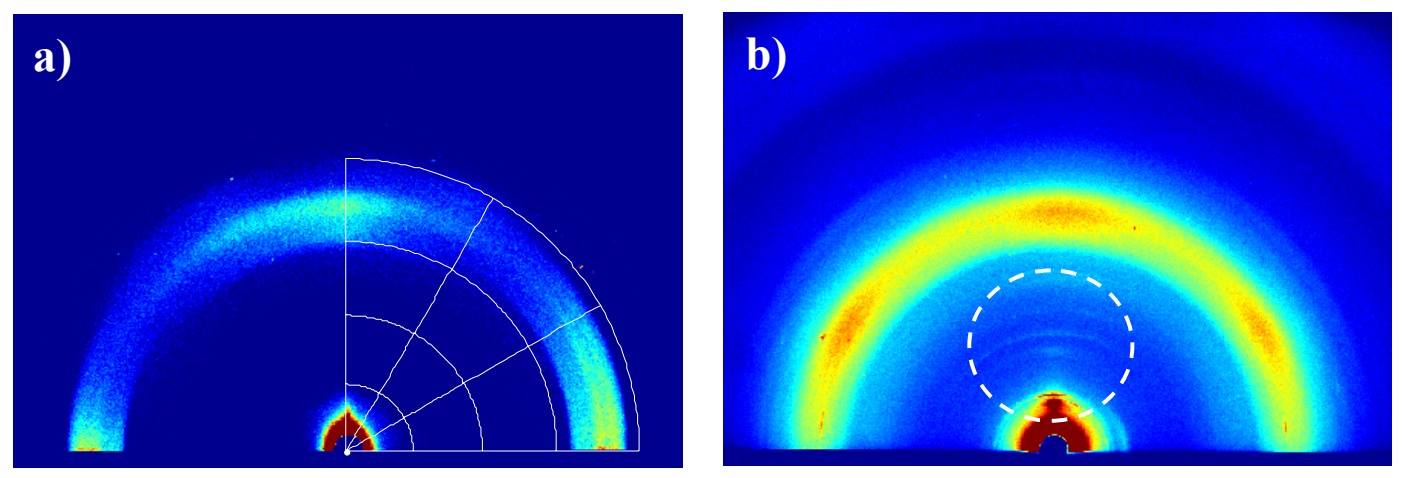

Figure 2 GIWAXS images, measured with $\mathbf{k}_{\text {in }}$ a) perpendicular, and b) parallel to the rubbing direction. The arcs in a) indicate $Q$ values of $0.5,1.0,1.5$ and $2.0 \AA^{-1}$. Note the qualitative differences between the images, with a 2-fold symmetry in (a) and 6-fold in (b). The slight asymmetry in a) is ascribed to sample misalignment. The broken line in b) encircles scattering features ascribed to the lamellae stacking of PEDOT;PSS. The region near the beamstop in these images corresponds to the small-angle (GISAXS) signal.

GIWAXS images obtained with the incoming beam parallel and perpendicular to the rubbing direction are qualitatively different, see Fig. 2, signifying preferred orientation of the atomic scale structure. Furthermore, from the angle dependent intensity distribution on the diffraction circles, we can conclude that the particles indeed have a preferred $\chi$-orientation, as suggested by the contrast differences in the TEM data. Thus, these images clearly show that the particles are biaxially oriented in the sample, i.e., two axes are needed to describe the (average) orientation of the particles. The nanorods have a preferred orientation of the hexagonal $c$-axis along the rubbing direction, and the $a-c$ (or, equivalently, $b-c$ ) plane parallel to the sample surface plane. The 6-fold intensity distribution around the 
$y$-axis (cf. Fig. 1 for definition of axes), combined with a 2-fold intensity distribution around the $x$-axis, is strong evidence for a hexagonal (wurtzite) unit cell.

Results from synchrotron GIWAXS measurements are shown in Fig. 3, with both radial scans along $Q_{\text {x }}$ and $Q_{\mathrm{y}}$, and "rocking scans" performed by rotating the sample about the film normal (motor $\varphi$ ). As expected from the above discussion, the sample anisotropy makes the $Q_{\mathrm{x}}$ and $Q_{\mathrm{y}}$ scans very different. Small features around $0.4 \AA^{-1}$ (not shown) and the broad shoulder at $1.3 \AA^{-1}$ in the $Q_{\mathrm{x}}$ - and $Q_{\mathrm{y}}$-scans can be ascribed to PEDOT:PSS. ${ }^{41}$ The in-plane anisotropy is highlighted in the in-plane rocking scans, shown for the 100 and 020 reflections, exhibiting a Gaussian intensity distribution with $W_{\varphi} \sim 52^{\circ}$ (fwhm). These $\varphi$-scans show a significant alignment of the hexagonal $c$-axis along the rubbing direction, since 002 exhibits peaks at $\varphi=0^{\circ}$ and $180^{\circ}$ (when $\mathbf{Q} \| \mathbf{y}$ ), and the 100 reflection has peaks at $\varphi= \pm 90^{\circ}$ (when $\mathbf{Q} \perp \mathbf{y}$ ). As required, the $<100>$ direction is at $90^{\circ}$ with respect to $<002>$. This supports the conclusion from the rotating anode studies shown in Fig. 2 that the nanorods exhibit a strong tendency of orienting with [001] (hexagonal $c$-axis) parallel to the rubbing direction. It is instructive to consider qualitatively the relative peak widths in Fig. 3(a) and (b). For example, the 002 reflection (at $Q$ $\left.=1.79 \AA^{-1}\right)$ is particularly narrow, consistent with the $c$-axis coinciding with the long axis of the nanorods.

Figure 3 also shows the manually fitted intensity for oriented CdSe nanoparticles of pure wurtzite structure, calculated using the outlined formalism and superposed on a smooth "background" curve accounting for both diffuse scattering and signals from the PEDOT polymer. The fits shown are for pure wurtzite structure. Zinc blende structures proved inadequate, and also various mixed-layer models including strain and stacking faults, ${ }^{3,28}$ did not further improve the appearance of the fits. The fits shown were obtained using a "superellipsoid" governed by the equation $\left(x / r_{\mathrm{a}}\right)^{2}+\left(y / r_{\mathrm{b}}\right)^{2}+\left(z / r_{\mathrm{c}}\right)^{4} \leq 1$, with $r_{\mathrm{a}}=r_{\mathrm{b}}=40 \AA$ and $r_{\mathrm{c}}=110 \AA$. The exponent of 4 implies a slightly more rectangle-shaped contour for cross sections parallel to the rod's long axis. This parameterization was chosen because it gives a reasonable description of the shapes observed by TEM. The resulting particle contained 40809 atoms, with the unit cell $c$-axis parallel to the rubbing direction $(\hat{\mathbf{y}})$, and with the $a-c$ plane parallel to the 
substrate, in accordance with the experimental observations. The simulations presented were thus made using Debye-Waller factors $\sigma=0.115$, which gives $\left\langle u^{2}\right\rangle \approx 0.04 \AA^{2}$, in line with previous reports. ${ }^{10}$ It is readily seen from the experimental curves of Fig. 3 that the simulation procedure gives good fits.

A final source of complication is that, not unexpectedly, the degree of out-of-plane orientation turned out to be different for the two orthogonal in-plane scans. This can be easily visualized by considering that it is intuitively easier for the elongated nanoparticles to roll about their long axis, than to tilt the long axis with respect to the substrate plane. This effect was incorporated by using different values $W_{\chi \perp}$ and $W_{\chi_{\|}}$for the parameter $W_{\chi}$ in the simulations of Fig. $3 \mathrm{a}$ and $3 \mathrm{~b}$, respectively. The other parameters, including the intensity scaling factor, were kept equal for the two simulations. The model parameters are listed in Table 1. For the instrumental broadening, $W_{\mathrm{Q}}$, a reasonable value of $0.03 \AA^{-1}$, ${ }^{35}$ was obtained by trial and error as part of the fitting procedure.

Table 1. List of parameters used for the simulations, cf. text for further explanations. The dependence on the lineshape parameters is weak.

\begin{tabular}{|l|l|l|}
\hline Parameter & Value & Comments \\
\hline$W_{\mathrm{Q}}$ & $0.03 \AA$ & Instrumental broadening \\
\hline$W_{\varphi}$ & $52^{\circ}(\mathrm{fwhm})$ & Cf. Fig. 3c. \\
\hline$W_{\chi \perp}$ & $58^{\circ}(\mathrm{fwhm})$ & \\
\hline$W_{\chi_{\|}}$ & $18^{\circ}(\mathrm{fwhm})$ & \\
\hline$\mu_{\mathrm{Q}}$ & 0.8 & $\sim$ Lorentzian lineshape \\
\hline$\mu_{\varphi}$ & 0 & Gaussian lineshape \\
\hline$\mu_{\chi}$ & 0 & Gaussian lineshape \\
\hline$r_{\mathrm{a}}$ & $40 \AA$ & Nanoparticle axes \\
\hline$r_{\mathrm{b}}$ & $40 \AA$ & \\
\hline$r_{\mathrm{c}}$ & $110 \AA$ & \\
\hline$\sigma$ & $0.115 \AA$ & Reported bulk value \\
\hline
\end{tabular}



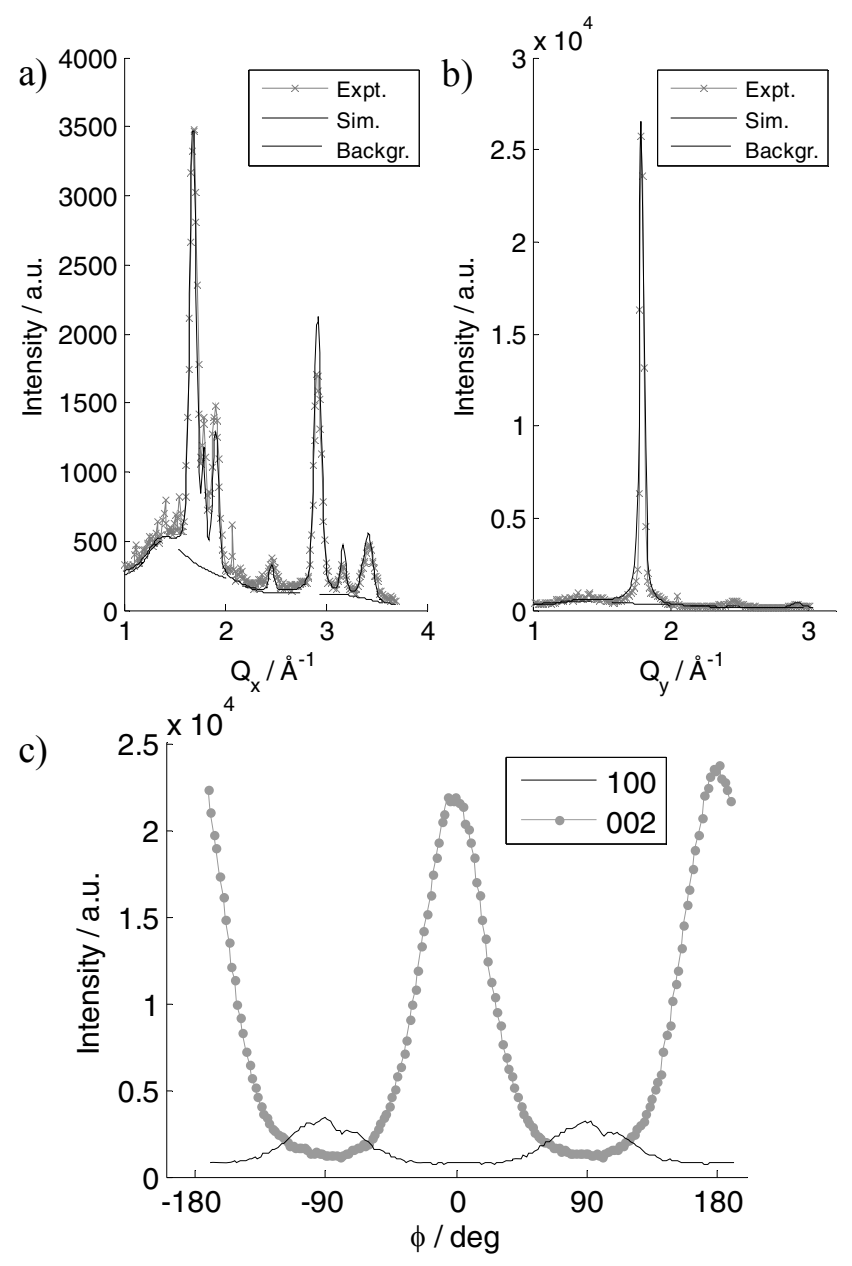

Figure 3: Synchrotron GIWAXS data and simulations, in-plane measurements. a) $Q_{x}$ scan (i.e., with $\mathbf{Q}$ perpendicular to the rubbing direction. b) In-plane $Q_{\mathrm{y}}$ scan, i.e., with $\mathbf{Q}$ parallel to the rubbing direction. c) In-plane "rocking curves" obtained for constant $Q$ by rotation about the sample normal (measured at $\left.Q_{\mathrm{z}}=0.035 \AA^{-1}\right)$, showing that the 002 reflection $\left(Q=1.79 \AA^{-1}\right)$ tends to orient along the rubbing direction $\left(\varphi=0^{\circ}\right)$, and $100\left(Q=1.69 \AA^{-1}\right)$ with $\varphi=90^{\circ}$. The peaks have the required $180^{\circ}$ periodicity, and are Gaussian with width $\sim 52^{\circ}$ (fwhm), being a direct measurement of the orientational distribution $W_{\varphi}$

The resulting fits are good, and the simplest possible model of a crude "average particle" consistent with all the experimental data is summarized in Fig. 4. By the chosen simulation approach, the X-ray diffraction peak positions, intensities and widths are simulated using only few (in the present case 11) 
physical model parameters. The approach taken here exploits the advantages of TEM and surface X-ray diffraction to reveal information about the CdSe particles. Because of the many factors contributing to diffraction signal, notably preferred orientation and anisotropic particle broadening, the partial complementarity between the methods is very useful for establishing the validity of the final model. However, to draw stronger conclusions regarding the Debye-Waller temperature dependence, disorder and strain effects, better experimental data is needed. Whereas the present X-ray data have the important advantage that they represent averages over a large number of particles $\left(\sim 10^{8}\right)$, it appears that more local techniques like convergent beam electron diffraction (CBED) and nano-focus X-ray diffraction, possibly combined with coherent scattering, are necessary to better understand the inner organization of chosen individual nanoparticles.

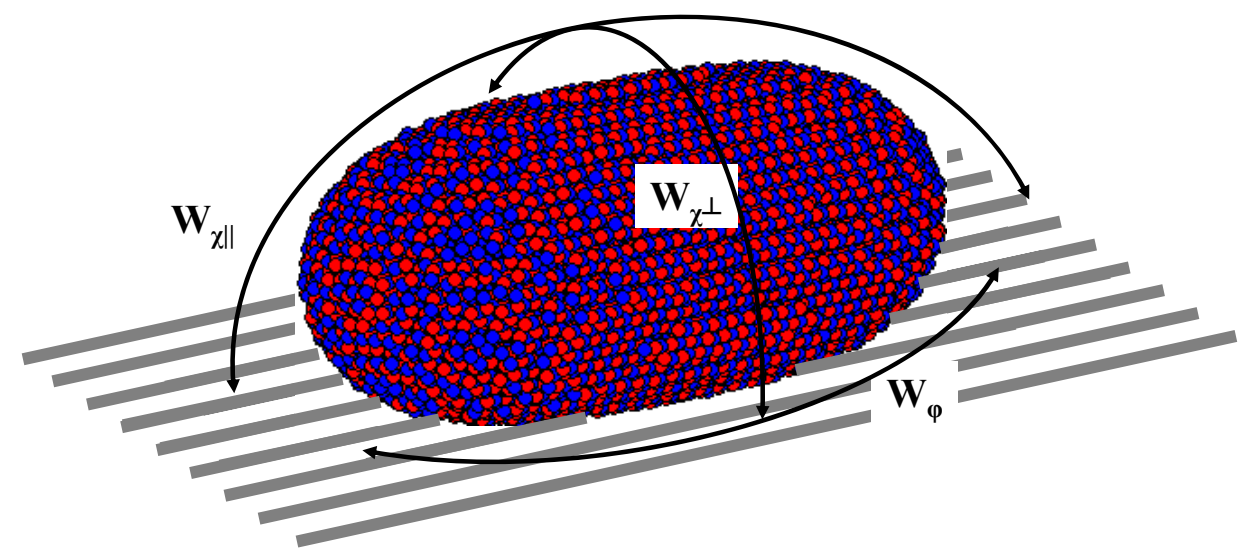

Figure 4. Illustration of the nanorod model, showing a "super-ellipsoid" orienting along the rubbing direction. The crystal structure is wurtzite, somewhat distorted by thermal disorder, with the $c$-axis parallel to the long axis of the particle, and an $a c$ (or equivalent) plane parallel to the substrate. The three angles used for describing the preferred orientation are indicated. The distribution is rather sharp in $W_{\chi_{\|}}$, with fwhm $\sim 18^{\circ}$, implying that the long axis strongly tends to lie down. For the two other axes, the distributions are significantly broader, with fwhm $\sim 52^{\circ}$.

CdSe nanoparticles have been studied using the complementary techniques of TEM, GISAXS and GIWAXS, combined with numerical simulations. TEM images provided direct information about the shape and size of the nanoparticles, which facilitated the construction of a realistic model of the 
particles. From the X-ray scattering and quantitative fits we conclude that the CdSe nanorods have the wurtzite structure, with the hexagonal $c$-axis coinciding with the long axis of the particles. The particles exhibit significant in-plane alignment with a distribution width $W_{\varphi} \approx 52^{\circ}$ (fwhm). There is also a significant preferred "rotational" orientation about the long axis of the nanorod. The preferred biaxial orientation is with the hexagonal $a$-c-plane parallel to the sample surface, and the particle long axis oriented along the rubbing direction of the sample. We have developed a simulation scheme that fits the experimental data well. The results obtained are of interest for further developments of e.g. polarized light sources. Moreover, the non-destructive methodology of grazing incidence X-ray scattering, which can be applied to thin film samples without elaborate sample preparations, is demonstrated to yield access to a wealth of structural information of the CdSe nanoparticles.

We thank Charles Darko and Christine M. Papadakis for assistance with the GISAXS measurements, and Emil J. Samuelsen for valuable discussions. Z.D. thanks the Graduate School of Excellence "Complex Materials at Interfaces" for financial support. The Norwegian Research Council, DanSync and NanoNed are gratefully acknowledged for financial support, and the HASYLAB staff for kind technical assistance.

\section{Supporting Information Available}

Full experimental details, GISAXS results, and further explanation of the calculation scheme. This information is available free of charge via the Internet at http://pubs.acs.org/. . 


\section{References}

1. $\quad$ Burda, C.; Chen, X. B.; Narayanan, R.; El-Sayed, M. A. Chem. Rev. 2005, 105, 1025-1102.

2. Manna, L.; Scher, E. C.; Alivisatos, A. P. J. Amer. Chem. Soc. 2000, 122, 12700-12706.

3. Aruguete, D. M.; Marcus, M. A.; Li, L. S.; Williamson, A.; Fakra, S.; Gygi, F.; Galli, G. A.; Alivisatos, A. P. J. Phys. Chem. C 2007, 111, 75-79.

4. Jadzinsky, P. D.; Calero, G.; Ackerson, C. J.; Bushnell, D. A.; Kornberg, R. D. Science 2007, 318, 430-433.

5. $\quad$ Bruchez, M.; Moronne, M.; Gin, P.; Weiss, S.; Alivisatos, A. P. Science 1998, 281, 2013-2016.

6. Chan, W. C. W.; Nie, S. M. Science 1998, 281, 2016-2018.

7. $\quad$ Shipway, A. N.; Katz, E.; Willner, I. ChemPhysChem 2000, 1, 18-52.

8. $\quad$ Gur, I.; Fromer, N. A.; Geier, M. L.; Alivisatos, A. P. Science 2005, 310, 462-465.

9. Huynh, W. U.; Dittmer, J. J.; Alivisatos, A. P. Science 2002, 295, 2425-2427.

10. Murray, C. B.; Norris, D. J.; Bawendi, M. G. J. Amer. Chem. Soc. 1993, 115, 8706-8715.

11. Peng, X. G.; Manna, L.; Yang, W. D.; Wickham, J.; Scher, E.; Kadavanich, A.; Alivisatos, A. P. Nature 2000, 404, 59-61.

12. Peng, Z. A.; Peng, X. G. J. Amer. Chem. Soc. 2001, 123, 1389-1395.

13. Bawendi, M. G.; Wilson, W. L.; Rothberg, L.; Carroll, P. J.; Jedju, T. M.; Steigerwald, M. L.; Brus, L. E. Phys. Rev. Lett. 1990, 65, 1623-1626.

14. Hu, J. T.; Li, L. S.; Yang, W. D.; Manna, L.; Wang, L. W.; Alivisatos, A. P. Science 2001, 292, 2060-2063.

15. Hikmet, R. A. M.; Chin, P. T. K.; Talapin, D. V.; Weller, H. Adv. Mater. 2005, 17, 1436-+.

16. Chin, P. T. K.; Hikmet, R. A. M.; Meskers, S. C. J.; Janssen, R. A. J. Adv. Funct. Mater. 2007, 17, 3829-3835.

17. Cohen, M. L., Chelikowsky, J.R., Electronic structure and optical properties of semiconductors. Springer Verlag: Berlin, 1988.

18. Tolbert, S. H.; Alivisatos, A. P. Science 1994, 265, 373-376.

19. Heaven, M. W.; Dass, A.; White, P. S.; Holt, K. M.; Murray, R. W. J. Amer. Chem. Soc. 2008, $130,3754-+$.

20. Zhu, M.; Aikens, C. M.; Hollander, F. J.; Schatz, G. C.; Jin, R. J. Amer. Chem. Soc. 2008, 130, 5883-+.

21. Huang, W. J.; Sun, R.; Tao, J.; Menard, L. D.; Nuzzo, R. G.; Zuo, J. M. Nature Mater 2008, 7, 308-313.

22. Bogan, M. J.; Benner, W. H.; Boutet, S.; Rohner, U.; Frank, M.; Barty, A.; Seibert, M. M.; Maia, F.; Marchesini, S.; Bajt, S.; Woods, B.; Riot, V.; Hau-Riege, S. P.; Svenda, M.; Marklund, E.; Spiller, E.; Hajdu, J.; Chapman, H. N. Nano Lett. 2008, 8, 310-316.

23. Dosch, H., Critical Phenomena at Surfaces and Interfaces. Springer Verlag: Berlin-Heidelberg, 1992.

24. Breiby, D. W.; Bunk, O.; Andreasen, J. W.; Lemke, H. T.; Nielsen, M. M. J. Appl. Cryst. 2008, 41, 262-271.

25. Breiby, D. W.; Hansteen, F.; Pisula, W.; Bunk, O.; Kolb, U.; Andreasen, J. W.; Mullen, K.; Nielsen, M. M. J. Phys. Chem. B 2005, 109, 22319-22325.

26. Breiby, D. W.; Samuelsen, E. J. J. Pol. Sci. B. Pol. Phys. 2003, 41, 2375-2393.

27. Hlawacek, G.; Shen, Q.; Teichert, C.; Resel, R.; Smilgies, D. M. Surf. Sci. 2007, 601, 25842587.

28. Gilbert, B.; Huang, F.; Zhang, H. Z.; Waychunas, G. A.; Banfield, J. F. Science 2004, 305, 651654.

29. Masadeh, A. S.; Bozin, E. S.; Farrow, C. L.; Paglia, G.; Juhas, P.; Billinge, S. J. L.; Karkamkar, A.; Kanatzidis, M. G., Phys. Rev. B 2007, 76, 115413.

30. Schmidt, T.; Clausen, T.; Falta, J.; Alexe, G.; Passow, T.; Hommel, D; Bernstorff, S. Appl. Phys. Lett. 2004, 84, 4367-4369. 
31. Levichev, S.; Chahboun, A.; Rolo, A. G.; Conde, O.; Gomes, M. J. M. Thin Solid Films 2009, 517, 2538-2540.

32. Knaapila, M.; Hase, T. P. A.; Torkkeli, M.; Stepanyan, R.; Bouchenoire, L.; Cheun, H. S.; Winokur, M. J.; Monkman, A. P. Cryst. Growth Des. 2007, 7, 1706-1711.

33. Breiby, D. W.; Bunk, O.; Pisula, W.; Solling, T. I.; Tracz, A.; Pakula, T.; Mullen, K.; Nielsen, M. M., J. Amer. Chem. Soc. 2005, 127, 11288-11293.

34. Naudon, A.; Thiaudiere, D. J. Appl. Cryst.1997, 30, 822-827.

35. Breiby, D. W.; Lemke, H. T.; Hammershoj, P.; Andreasen, J. W.; Nielsen, M. M. J. Phys. Chem. C 2008, 112, 4569-4572.

36. Als-Nielsen, J., Elements of Modern X-ray Physics. Wiley-VCH: New York, 2001.

37. Smilgies, D. M. Rev. Sci. Instr. 2002, 73, 1706-1710.

38. Vetelino, J. F.; Gaur, S. P.; Mitra, S. S. Phys. Rev. B 1972, 5, 2360-\&.

39. Warren, B. E., X-ray Diffraction. Addison-Wesley Pub. Co.: Reading, Mass., 1969.

40. Li, L. S.; Walda, J.; Manna, L.; Alivisatos, A. P. Nano Lett. 2002, 2, 557-560.

41. Breiby, D. W.; Samuelsen, E. J.; Groenendaal, L.; Struth, B. J. Pol. Sci. B-Pol. Phys. 2003, 41, (9), 945-952.

TOC Graphic

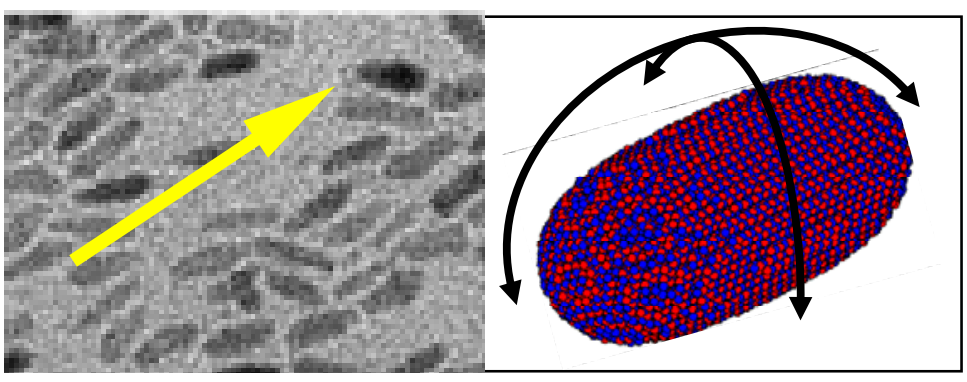

